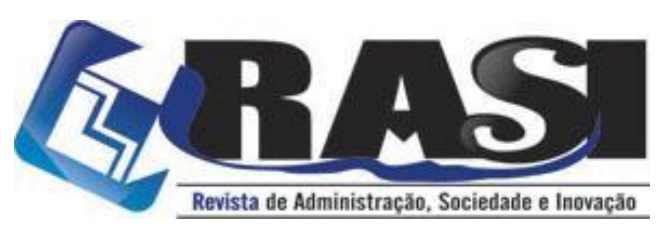

http://www.rasi.vr.uff.br

RASI, Volta Redonda/RJ, v. 8, n. 1, pp. 07-22, Jan./Abr. 2022

\title{
Análise das práticas de logística reversa (LR) na região Sul Fluminense, após a implementação da Política Nacional de Resíduos Sólidos (PNRS)
}

\author{
Janinne da Silva Bitencourt (UFF) - janinnebitencourt@id.uff.br \\ Cecilia Toledo Hernández (UFF) - ctoledo@id.uff.br
}

\begin{abstract}
Resumo:
A Política Nacional de Resíduos Sólidos (PNRS) é um marco brasileiro em relação a gestão dos resíduos sólidos. Neste contexto, a legislação, não somente a brasileira, se torna cada vez mais rigorosa em relação ao descarte de produtos no final de seus ciclos de vida. Este artigo utilizou o critério de Leite (2012) que classificava os programas em econômicos, de serviço ao cliente, legais, de cidadania corporativa e de imagem corporativa. Neste sentido, paira a dúvida se realmente o programa legal está sendo considerado importante nas empresas. Com base neste contexto, o objetivo da pesquisa é avaliar a importância de motivadores da logística reversa (LR) em empresas da Região Sul Fluminense, no ramo automobilístico, após a implementação da PNRS. O estudo tem caráter aplicado e exploratório e a coleta de dados foi feita por meio de questionários. A fim de analisar as informações obtidas, utilizou-se o método Analytic Hierarchy Process (AHP). Nesta pesquisa, o programa econômico foi considerado o mais importante seguido do programa legal. Outro estudo feito na Região Sul Fluminense também teve como destaque o programa econômico. Entretanto, em grau de priorização, o programa legal não foi considerado um motivador de LR, sendo que na atual pesquisa este se encontra em segundo lugar em termos de importância. Como recomendação de pesquisa futura, a análise pode ser feita em outras regiões e ramos.

Palavras-Chave: Economia Circular; Logística Reversa; Multiple Criteria Decision Making (MCDM); Política Nacional de Resíduos Sólidos (PNRS).

\section{Analysis of reverse logistics practices (LR) in the South Fluminense region, after the implementation of the National Solid Waste Policy (PNRS)}

\begin{abstract}
:
The National Solid Waste Policy (PNRS) is a Brazilian landmark in relation to solid waste management. In this context, legislation, not only the Brazilian one, becomes increasingly strict regarding the disposal of products at the end of their life cycles. This article uses Leite's (2012) criteria, which classifies programs in economics, customer service, legal, corporate citizenship, and corporate image. In this sense, the question remains whether the legal program is really being considered important in companies. Based on this context, the objective of the research is to evaluate the importance of reverse logistics (RL) motivators in companies in the South Fluminense region, in the automobile sector, after the implementation of the PNRS. The study has an applied and exploratory character and data collection was done through questionnaires. To analyze the information, the Analytic Hierarchy Process (AHP) method was used. In this research, the economic program was considered the most important followed by the legal program. Another study carried out in the South Fluminense region also highlighted the economic program. However, in terms of prioritization, the legal program was not considered a RL motivator, and in the current research it is ranked second in terms of importance. As a recommendation for future research, an analysis can be done in other regions and branches.
\end{abstract}

Keywords: Circular Economy; Reverse logistic; Multiple Criteria Decision Making (MCDM); National Solid Waste Policy (PNRS).

$\begin{array}{r}\text { R. Desembargador Ellis Hermydio Figueira, 783, Bloco A, sl. 218, Aterrado. } \\ 27213-415 \text { - Volta Redonda, RJ - Brasil } \\ \text { www.uff.br } \\ \hline \underline{\text { Fiversidade }} \\ \hline \begin{array}{c}\text { Copyright (C) 2020 RASI. Todos os direitos, até mesmo de tradução, são reservados. É } \\ \text { permitido citar parte de artigos sem autorização prévia, desde que seja identificada a fonte. }\end{array} \\ \hline\end{array}$




\section{Análise das práticas de logística reversa (LR) na região Sul Fluminense, após a implementação da Política Nacional de Resíduos Sólidos (PNRS).}

\section{Introdução}

Temáticas relacionadas a sustentabilidade adquirem notório destaque com a atual propensão de crescimento populacional, com maiores montantes de desperdícios, derivados de processos industriais, poluição da água, do ar ou da terra e com uma crescente consciência ambiental na sociedade, devido a legislações mais rigorosas e produtos com menores ciclos de vida (Bhandari et al., 2019).

Neste contexto, vários assuntos como a logística reversa (LR) e a economia circular (EC) vem sendo discutidos. Alguns estudos buscam tornar mais claro o conceito de economia circular ao fazer uma revisão sistemática da literatura (Farooque et al., 2019; Geisendorf \& Pietrulla, 2018; Geissdoerfer et al., 2017). Nestes estudos, é mostrada uma gradativa preocupação ambiental ao redor do mundo, e muitos destes destacam a China, como um país que mostra uma progressiva preocupação com a temática em diversos ramos da economia (Geng et al., 2012; Ness, 2008; Su et al., 2013). No cenário brasileiro, a Política Nacional de Resíduos Sólidos (PNRS), sancionada em 2010, deu grande notoriedade a EC, pois esta marca a gestão dos resíduos sólidos no Brasil (Oliveira et al., 2019).

Percebe-se em todo este novo cenário, que o conceito de LR se relaciona com a EC, sendo um de seus princípios e sua relevância aumenta à medida que ela conceitua e clarifica a gestão de retorno de produtos. Desta maneira, uma parte crítica do gerenciamento das organizações é o fluxo reverso, tratado como um fator estratégico. Na perspectiva brasileira, ainda há a necessidade de maior disseminação do conceito da EC, pois falta divulgação local e nacional do modelo, o que ainda coloca o país em posição inferior de pesquisas em relação a muitos países da Europa e da China (Oliveira et al., 2018).

Diante do ainda estágio inicial de pesquisa nestes assuntos no cenário nacional, o artigo buscou responder a seguinte questão: Quais são os programas de LR priorizados após a PNRS no ramo automobilístico? Desta maneira, o objetivo geral desta pesquisa é avaliar programas e atividades neste ramo, relacionados à LR na região Sul Fluminense, através do método de auxílio multicritério a decisão, Analytic Hierarchy Process (AHP). Entretanto, a pesquisa se delimita espacialmente, pois analisa somente empresas de uma região específica.

A LR é importante para o fortalecimento das empresas, porém ainda existem muitas barreiras na implementação da LR, especialmente no ramo automobilístico (Lima, 2016; Vieira et al., 2020). As principais barreiras no contexto brasileiro, são internas, como econômicas, de infraestrutura e gestão das empresas e externas as organizações, como falta de controle e fiscalização em relação a legislação. Desta maneira, conhecer as práticas de LR no ramo automobilístico é importante para uma melhor gestão do fluxo reverso, tanto para os gestores privados e públicos, no sentido de analisar se os programas legais vêm ganhando notoriedade e quais necessitam de maior atenção. Assim, estruturar um canal de fluxo reverso é relevante por questões ambientais, bem estar social e no aspecto econômico, uma vez que pode gerar ganhos de competividade nos produtos nacionais (Lima, 2016).

Este trabalho se estrutura em seis seções, sendo a seção 2, uma fundamentação teórica sobre a economia circular e especificamente sobre a LR, a seção 3, um tratamento das questões metodológicas da pesquisa, a seção 4, uma exposição dos resultados obtidos com a utilização do método AHP, a seção 5, a discussão dos resultados e a seção seis, uma 
apresentação das principais conclusões e sugestões de trabalhos futuros, seguidos das referências bibliográficas.

\section{Referencial Teórico}

A fundamentação teórica tem como objetivo permitir a análise do tema objeto de estudo. Para isto, foram analisados diferentes aspectos sobre o tema de pesquisa como a sustentabilidade, a economia circular e lei de resíduos sólidos no Brasil, a LR e sua relação com a simbiose industrial, além de programas de LR e o uso da tomada de decisão com múltiplos critérios.

\subsection{Sustentabilidade}

Dentro da temática da sustentabilidade, caracterizada como o processo pelo qual a atividade humana minimamente influencia o ecossistema terrestre, uns dos principais conceitos sustentáveis é o tripé da sustentabilidade que estabelece três dimensões para as organizações, a econômica, a ambiental e a social (Batista et al., 2018).

A dimensão econômica se concentra em construir capital financeiro. Gerar capital social preocupar-se com a responsabilidade interna ou externa das empresas, caracteriza a dimensão social da sustentabilidade. A dimensão ambiental teria como objetivo, minimizar o uso de recursos naturais (Elkington, 1998).

Dentro desta temática, alguns conceitos emergiram e são semelhantes a EC, apesar de possuírem algumas diferenças entre si, como o conceito "cradle to cradle", as cadeias de suprimentos fechadas, a ecologia industrial, a LR, que será estudada neste artigo, e a biomimética (Geisendorf \& Pietrulla, 2018).

Dentro do contexto sustentável, a principal definição de economia circular é dada pela fundação Ellen MacArthur Foundation (2015), que a define como um sistema restaurativo e regenerativo intencionalmente (Farooque et al., 2019; Geisendorf \& Pietrulla, 2018). Outra famosa definição se dá ao contrapor-se a economia linear, modelo de "pegar, fazer e descartar", pois, este modelo é ineficiente em relação ao tripé da sustentabilidade (Ghisellini et al., 2016; Ness, 2008).

Percebe-se com o tripé da sustentabilidade, que implementar o desenvolvimento sustentável nas empresas não é de simples operacionalização. Assim, é necessário para isto, mudanças na tomada de decisão e no modo de produção e consumo (Lang et al., 2012). Para enfrentar todas estas barreiras da sustentabilidade, Escrig-Olmedo et al. (2019), expuseram a necessidade de uma ampla geração de conhecimento em relação aos principais princípios da sustentabilidade e a interação entre diferentes gerações (a passada, a presente e a futura). Para vencer os desafios e gerar maior conhecimento no campo da sustentabilidade, o desenvolvimento sustentável deve ser operacionalizado, surgindo para isto alguns conceitos mais estruturados como a EC e a LR (Geissdoerfer et al., 2017).

\subsection{Economia Circular e Lei de Resíduos Sólidos no Brasil}

O conceito de EC é relativamente novo e teve sua definição proposta por Boulding (1966) com o livro The Economic of the Coming Spaceship Earth (Geisendorf \& Pietrulla, 2018). Adicionalmente, a EC tem diferentes níveis de implementação ao redor do mundo (Ghisellini et al., 2016).A China, em relação a implementação da EC, se destaca mundialmente com vários estudos nesta área de estudo (Wang \& Wang, 2020).Parte desta grande influência chinesas e deve especialmente a promoção da Lei de Economia Circular, 
homologada em 2008 que impulsiona o fechamento de processos produtivos diversos (Machado et al., 2012).

Nos Estados Unidos, a Agência de Proteção Ambiental dos Estados Unidos (EPA) é parte essencial da implementação da EC, uma vez que regulariza a gestão de resíduos domésticos, perigosos ou não, com base na lei de Conservação e Recuperação de Recursos (RCRA) (EPA, 2002). Na União Europeia, há uma diretiva que regula a gestão de resíduos, a diretiva 2008/98/EU, responsável por reutilizar, reciclar e recuperar energia.

Em relação ao cenário brasileiro, o Brasil ainda precisa ter uma maior disseminação do conceito da EC, uma vez que falta divulgação local e nacional do modelo, o que coloca o país ainda em posição inferior de pesquisas em relação a muitos países da Europa e da China (Oliveira et al., 2018).

A EC possui uma grande importância no mundo e no Brasil para se implantar processos sustentáveis e de acordo com a sua mais famosa definição, dada por Ellen MacArthur Foundation (2015), a EC é um sistema restaurador e regenerativo, que percebe os produtos ao final de sua vida útil, não como resíduos, assim como no sistema linear, mas como recursos. Assim, para efetiva implementação do sistema circular, destaca-se a gestão de resíduos como um dos principais processos do conceito.

A PNRS foi o marco na gestão dos resíduos sólidos no Brasil, pois preencheu uma lacuna na legislação brasileira ambiental baseado na gestão internacional e suas legislações. Esta política brasileira destaca a responsabilidade compartilhada pelo ciclo de vida do produto, acordos setoriais e LR (GRS-UFPE, 2014). A responsabilidade compartilhada envolve todos os envolvidos no ciclo de vida dos produtos, os fabricantes, importadores, distribuidores e comerciantes que devem se preocupar com a destinação final e o impacto do resíduo, sendo a LR fundamental neste processo (CNI, 2019).

Entretanto, a PNRS enfrenta muitos desafios em sua implementação ao longo do país. Falta de fundos governamentais, falta de capacidade técnica e administrativa, falta de conscientização do público em relação à geração de resíduos e insuficiência da estratégia de descentralização do governo federal na implementação da agenda (Cetrulo et al., 2018).

\subsection{Logística Reversa (LR)}

De acordo com o Council of Supply Chain Management Professionals, a LR é uma área especializada da logística, que foca no movimento de produtos após a venda, incluindo os produtos que são devolvidos. No que tange ao aumento da conscientização, em relação a temáticas sustentáveis e as necessidades dos clientes cada vez mais inovadores, a LR se tornou tão importante quanto a logística em seu fluxo direto (Vlachos, 2016). Assim, alguns dos motivadores principais para se implementar a LR nos diversos processos produtivos são fatores financeiros, necessidades dos clientes e legislações mais rigorosas (Vlachos, 2016).

Alguns autores buscaram relacionar a LR como um pilar da EC, de modo a destacar a importância que a LR tem na implementação do modelo circular. Estes dois conceitos são tidos como semelhantes, porém o conceito de EC é mais amplo, uma vez que não cobre só o lado reverso nas cadeias de suprimentos (Beiler et al., 2020). Ao relacionar os conceitos de EC e LR, os ramos da economia mais estudados nesta relação de conceitos são o da construção civil, de embalagens, de reciclagem, incluindo os catadores, do poliestireno, comida e bebida, ramo automobilístico e de equipamentos elétricos eletrônicos. Ainda são poucas as publicações em EC separadamente da LR ao perceber mais trabalhos que se concentram em estudar somente a LR, e não como ela se inclui na EC (Cosenza et al., 2020). 
O conceito mais difundido e que se insere na ecologia industrial (EI), é a simbiose industrial (SI), que é essencial para fechar os ciclos produtivos, ou seja, é de grande importância na implementação da LR. A EI se baseia na comparação da natureza e do ambiente industrial (Trevisan et al., 2016). Já a SI é definida como a troca que ocorre entre materiais, energia, água e coprodutos (Ferreira et al., 2021). Assim, como a LR, a SI destaca a importância da colaboração entre os diferentes stakeholders, sejam eles, funcionários e gestores privados, sociedade, governo (Mota \& Paula, 2020; Pinheiro et al., 2020). Assim, é essencial que o setor público e privado crie um ambiente que seja propício para a prática da SI e LR.

Além destes conceitos que se relacionam de alguma forma, motivadores da LR, barreiras e aplicação de métodos de tomada de decisão em alguns ramos específicos da economia são alguns dos principais temas estudados por Abbas e Farooquie (2020) e Agrawal e Singh (2019).

\subsection{Programas de LR: Motivadores e Barreiras}

Razões econômicas, ambientais, legislação e necessidade de se destacar no mercado competitivo são alguns dos motivadores para se implementar a LR. Entretanto, muitas são as barreiras desta implementação (Brito \& Dekker, 2004). Demajorovic et al. (2012), destacam a importância de motivadores econômicos, de imagem e de serviço ao cliente. Couto e Lange (2017) definem alguns aspectos importantes, como legislação e normatização, aspectos tributários e financeiros, controle governamental e participação da população, para se implementar a LR no contexto brasileiro.

Realizou-se uma pesquisa bibliográfica a partir das bases de dados bibliográficas científico-técnicas, Web of Science e Scopus, após a homologação da PNRS, em 2010. As palavras-chaves contidas no título, palavras-chaves ou resumo utilizadas para recuperar os artigos são: Reverse Logistics, Drivers ou Reverse Logistics Programs ou Reverse Logistics Barriers. Isso resultou em mais de 60 artigos. Foi realizado um processo de triagem, em que os títulos e resumos foram analisados para assegurar que seu tema principal estava adequado ao escopo da pesquisa. O conjunto final de artigos usados neste estudo para identificar as práticas de LR foram de 35 artigos.

Percebe-se que os diferentes motivadores de LR, faz com que exista diferença nos objetivos dos programas estabelecidos por alguns autores. Adicionalmente, com esta pesquisa bibliográfica, percebeu-se que todos os autores classificavam seus programas seguindo a lógica dos cinco programas, estudados por Leite (2012), Hernández (2010), Hernández et al. (2012), Mangla et al. (2012), Eltayeb et al. (2010), Govindan e Bouzon (2018) e Kumar e Putnam (2008), definem programas econômicos, de serviço ao cliente, legais, de cidadania corporativa e de imagem corporativa, assim como Leite (2012).

\section{Método}

A pesquisa pode ser classificada como aplicada quanto a sua natureza, ou seja, tem por objetivo gerar conhecimentos para uma aplicação prática ao solucionar problemas específicos (Gerhardt \& Silveira, 2009). No caso deste artigo, a pesquisa terá aplicação prática ao analisar motivadores de LR na região Sul Fluminense.

$\mathrm{Na}$ classificação quanto aos objetivos, seu caráter é exploratório uma vez que tem por finalidade fazer com que o problema se torne mais explícito (Gil, 2008). A pesquisa pode ser definida como qualitativa, pois os dados são coletados segundo uma escala qualitativa e toda 
a análise numérica posterior se dá a partir desta. O estudo seguiu os quatro passos apresentados na Figura 1.

Figura 1. Etapas da Pesquisa

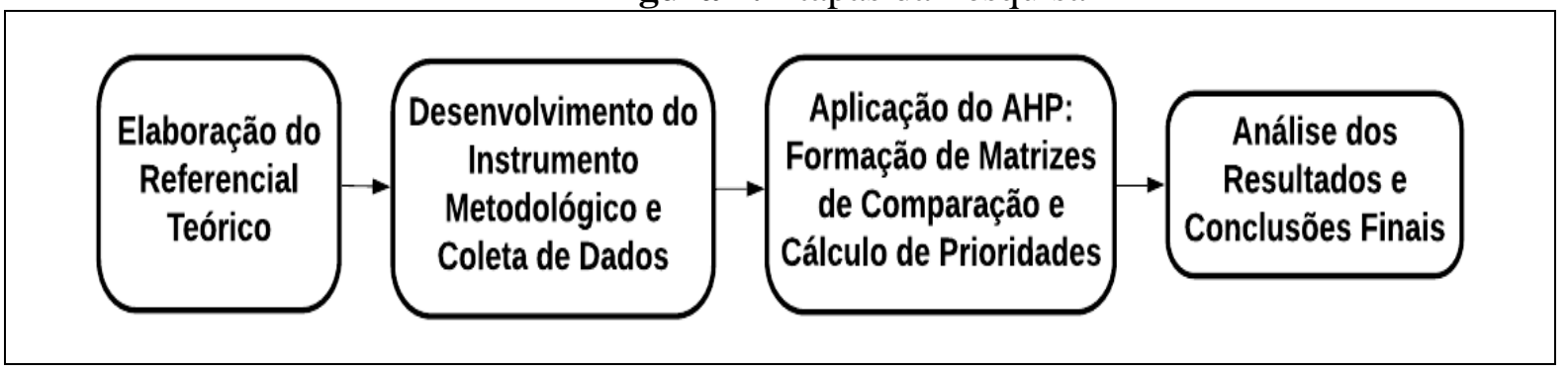

Fonte: elaboração própria (2021).

Além da classificação da pesquisa, uma etapa importante da metodologia é a descrição dos procedimentos metodológicos e o seu desenvolvimento. Segundo Creswell (2007), uma etapa importante no procedimento de pesquisa é o instrumento de coleta e análise de dados.

O instrumento de coleta de dados foi o questionário, baseado em Hernández (2010), que contava com questões abertas em uma primeira fase e com questões fechadas, para julgamentos da análise multicritério, em uma segunda fase. $\mathrm{O}$ objetivo inicial, para $\mathrm{o}$ levantamento qualitativo, foi a obtenção de informação abrangente sobre as características dos programas e atividades de LR. A segunda fase é aquela na qual os profissionais de LR definiram quais programas e atividades estão presentes em suas empresas.

No caso deste artigo, a amostra foi selecionada com um professor do curso de especialização de um centro universitário de Volta Redonda, de maneira presencial. Esta amostra contou com 3 profissionais especializados na área de logística, que representaram três montadoras automobilísticas importantes da Região Sul Fluminense. Deste modo, a amostragem foi feita por conveniência e consome pouco tempo quando comparada com outras técnicas

Segundo Jonathas e Paiva (2020), o automobilístico nesta região conta com cinco montadoras, MAN Latin America, PSA Peugeot Citroën, Renault-Nissan, Hyundai e Jaguar Land Rover. Apesar dos especialistas da amostra serem acessíveis e dispostos a cooperar, uma das desvantagens desta coleta foi a existência de vieses nas respostas, de maneira a utilizar práticas de LR destacados na literatura para melhor aproveitamento dos questionários.

Adicionalmente, a amostra foi formada somente por especialistas da região Sul Fluminense, uma vez que o panorama depois da homologação da PNRS, exposto neste artigo, será comparado com panorama anterior explicitado pelo estudo de Hernández (2010) que também utilizou a região Sul Fluminense como referência.

Para definir os programas e atividades de LR que comporiam o questionário, foi utilizado o critério de Leite (2012). Segundo classificação do autor, o programa econômico visa otimizar a obtenção de lucros, o de serviço ao cliente visa destacar a empresa no mercado e diferenciá-la quanto aos serviços prestados aos clientes, o legal visa cumprir com as legislações existentes, o de cidadania corporativa responsabiliza as organizações como socialmente responsáveis e o de imagem tem como objetivo reforçar a imagem das empresas no mercado.

Nestes programas, foram definidas atividades de LR que são realizadas de acordo com modelo proposto por Hernández (2010) na Tabela 1. 
Tabela 1. Programas e Atividades de LR

\begin{tabular}{ll}
\hline Programas de LR & Atividades ou alternativas de LR \\
\hline & -Materiais retornados ao processo produtivo. (A1) \\
& -Reuso de embalagens e venda como matéria-prima para outros processos. (A2) \\
& -Revenda de produtos em mercados secundários. (A3) \\
Programas Econômicos & -Reciclagem. (A4) \\
(PE) & -Existência de registro dos custos gerados pelas devoluções. (A5) \\
& -Desembolso por ações sociais e meio ambientais. (A6) \\
& -Despesas por treinamento de funcionários. (A7) \\
& -Custos para operar o canal reverso (coleta, seleção, transporte, armazenagem). (A8) \\
& -Custos para desenvolver novas tecnologias. (A9) \\
\hline Programas de Imagem (PI) & -Propaganda como empresa responsável quanto aos seus produtos e processos. \\
& (A10) \\
& -Desenvolvimento de novas tecnologias para aproveitar os materiais reciclados. \\
& (A11) \\
-Destino adequado aos resíduos. (A12) \\
\hline Programas de Cidadania & -Projetos sociais. (A13) \\
Corporativa (PC & -Projetos educacionais. (A14) \\
& - Criação de emprego para operar o canal reverso. (A15) \\
\hline Programas de Serviço ao & -Parcerias com stakeholders. (A16) \\
Cliente (PS) & -Políticas de retorno liberais. (A17) \\
& -Fidelização de clientes. (A18) \\
ProgramasLegais (PL) & -Retornos bem definidos. (A19) \\
\hline & -Responsabilidade das empresas pelo destino correto de seus produtos no fim da \\
& -Estabelecimento de níveis mínimos de recuperação a serem cumpridos pelas \\
& empresas. (A21) \\
\hline &
\end{tabular}
Fonte: elaboração própria (2021).

Nota-se pela Tabela 1, que diferentes alternativas foram estabelecidas para os cinco programas de LR estabelecidos por Leite (2012).

Após a coleta de dados, foi medida a importância das práticas de LR, com o uso do AHP, devido a sua maior simplificação e conveniência. O AHP, um método de auxílio multicritério à decisão, foi desenvolvido por Saaty (1980) e tem como objetivo resolver problemas complexos, mas é considerada uma técnica de simples manejo.

O AHP é um dos métodos de auxílio multicritério à decisão utilizado para resolver problemas complexos, mas de simples manejo. Para aplicar o AHP foram seguidas as seguintes etapas baseadas em Saaty (2010):

1. Representação da hierarquia: O problema teve como objetivo analisar a priorização dos programas de LR na indústria automotiva. Para isto foi decomposto em dois níveis: critérios (programas de LR) e alternativas (atividades de LR desenvolvidas pelas empresas).

2. Comparação de pares: Comparação entre si e em pares de todos os critérios e das alternativas com respeito a cada critério. Para a construção destas matrizes de comparações pareadas, utilizou a escala de Saaty que utiliza uma escala de importância entre 1 e 9. Nesta escala, 1 significa que os elementos comparados contribuem de igual forma para o objetivo e na medida que os valores aumentam a importância de um com respeito ao outro também aumenta até o 9, onde um elemento tem importância absoluta sobre outro. 
3. Método dos autovalores: Nesta etapa, foram estimados os pesos dos elementos de decisão (peso dos critérios/programas e das alternativas/atividades), utilizando-se a síntese hierárquica.

4. Avaliação da consistência das comparações por pares: Foi calculado o índice de consistência das matrizes que não deve exceder 0,10 .

5. Agregação das prioridades: As prioridades relativas foram agregadas para se avaliar os resultados e desta forma obter um ranking dos critérios/programas e das alternativas/atividades através das prioridades globais.

Este último resultado com a prioridade global de critérios/programas de LR e alternativas/atividades de LR é analisado e comparado com estudo de Hernández (2010).

\section{Resultados}

Foi realizada a comparação par a par dos critérios, a fim de medir a importância de cada um dos programas de LR. Essa comparação, em formato de matrizes será apresentada e discutida nesta seção. De acordo com Lima (2016), estudos como este é fundamental para o debate sobre a implementação da LR no ramo automobilístico brasileiro, de modo a contribuir para o avanço do tema e para possíveis soluções que se adequem ao contexto brasileiro.

\subsection{Ramo Automobilístico}

Para a aplicação do método AHP, foi estruturada a matriz hierárquica da Figura 2. No primeiro nível, encontra-se o objetivo, seguido dos critérios do modelo e das alternativas. Os critérios são os programas de LR identificados pelos respondentes e as alternativas são as atividades de LR realizadas nas empresas analisadas.

Figura 2. Árvore hierárquica com os critérios e alternativas no ramo automobilístico

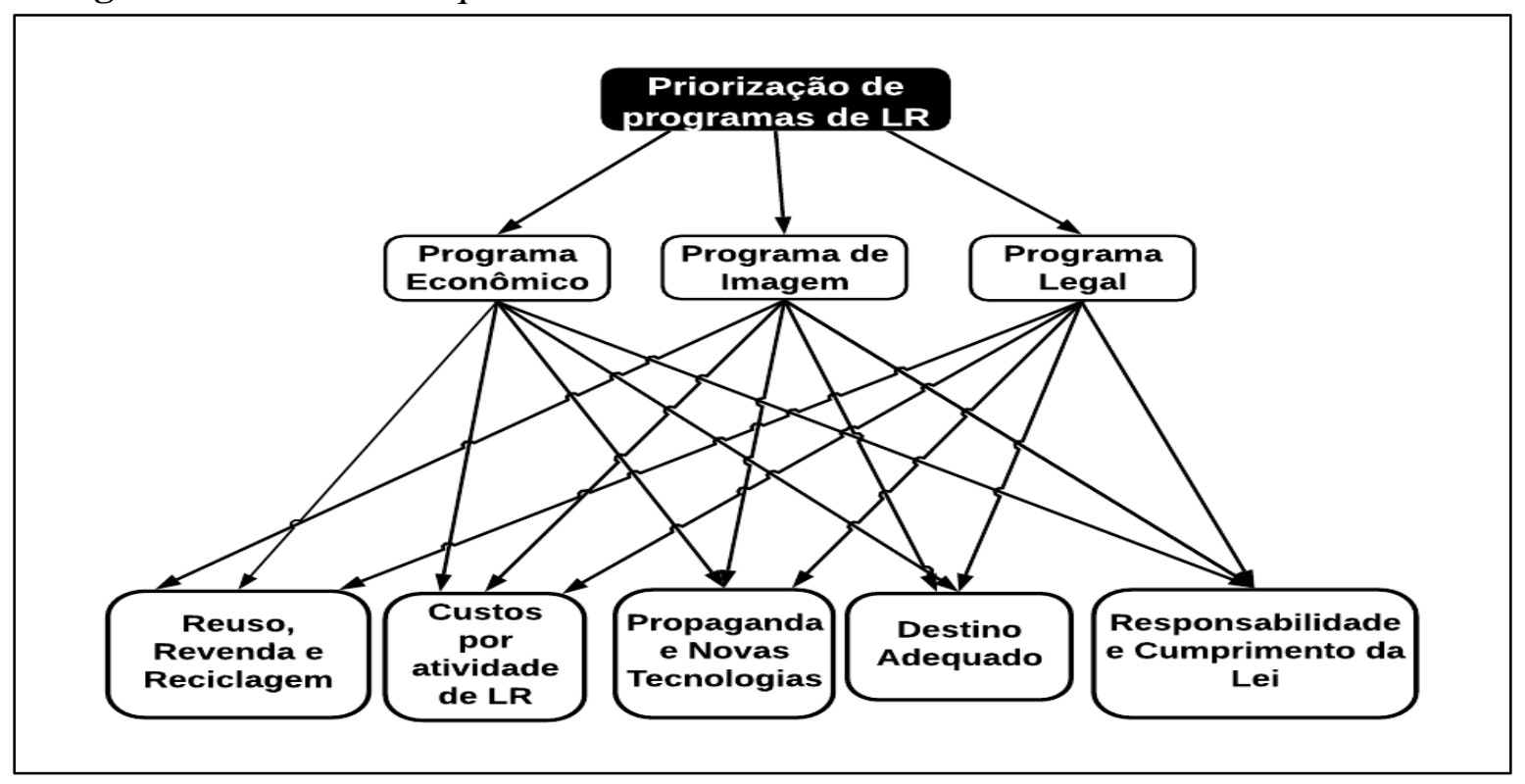

Fonte: elaboração própria (2021).

Como pode ser observado na Figura 2, no ramo automobilístico foram destacados 3 programas e 5 atividades 
Como etapa seguinte foram necessárias desenvolver 4 matrizes de comparação (uma matriz para a comparação dos critérios). A Tabela 2 mostra a comparação entre os critérios/programas de LR estabelecidos para o ramo automobilístico

Tabela 2. Matriz comparativa entre os critérios (Programas de LR)

\begin{tabular}{lccccc}
\hline Matriz Comparativa & PE & PL & PI & Vetor de prioridades \\
\hline Programa Econômico-PE & 1 & 4 & 9 & 0,71707 \\
Programa Legal-PL & & 1 & 4 & 0,21717 \\
Programa de Imagem-PI & & & & 1 & 0,06577 \\
\hline
\end{tabular}

Fonte: elaboração própria (2021).

Observa-se pela Tabela 2, que o vetor de prioridades obtido dita que o programa econômico tem uma prioridade maior em relação aos outros, seguido do programa legal.

Para verificar se o modelo é consistente, Saaty e Sodenkamp (2010), destacam a Taxa de Consistência (CR), que é determinado pela razão entre o Índice de Consistência e o Índice de Consistência Aleatório. A matriz será considerada consistente se a proporção resultante for menor que $10 \%$. Para a comparação dos critérios/programas de LR da Tabela 2, obteve-se um índice de consistência de 0,03548 , ou seja aproximadamente $3,55 \%$, valor menor que o valor de $10 \%$ ou 0,1 , o que mostra a consistência da matriz.

De igual forma foram elaboradas outras três matrizes que permitiram a comparação entre as alternativas/atividades de LR com respeito a cada critério/programa de LR. O resultado de cada vetor de prioridade é mostrado na Tabela 3.

Tabela 3. Prioridade de Cada Alternativa com Respeito aos Programas

\begin{tabular}{lccc}
\hline \multicolumn{1}{c}{ Matriz Comparativa } & PE & PL & PI \\
\hline Reuso, revenda e reciclagem- A2, A3, A4 & 0,29582 & 0,15070 & 0,08913 \\
Custos por atividade de LR- A8, A9 & 0,52842 & 0,15070 & 0,03601 \\
Propaganda e novas tecnologias- A10, A11 & 0,03254 & 0,02548 & 0,53418 \\
Destino adequado aos resíduos- A12 & 0,05914 & 0,02548 & 0,30376 \\
Responsabilidade e cumprimento da lei- A20, A21 & 0,08409 & 0,28560 & 0,03693 \\
\hline
\end{tabular}

Fonte: elaboração própria (2021).

Percebe-se pela Tabela 3, que:

- As atividades A8, A9, custos para operar e desenvolver novas tecnologias no canal reverso, são as mais destacadas ou prioritárias quando analisados os programas com objetivos econômicos.

- Quando avaliadas as atividades com respeito ao programa legal, as mais importantes são as A20 e A21, relacionadas à responsabilidade por cumprimento da lei.

- Com respeito ao programa de imagem, as atividades de maior peso são as relacionadas à propaganda e desenvolvimento de novas tecnologias, atividades A10 e A11.

$\mathrm{O}$ valor do CR para as matrizes ficou entre 0,02982 e 0,09739, menores que o valor 0,1, mostrando valores de CR consistentes.

Por último foi calculado o desempenho global de cada programa de LR no ramo automobilístico, além do desempenho de cada alternativa como mostrado na Tabela 4. 
Tabela 4. Prioridades dos programas de LR

\begin{tabular}{lc}
\hline Critérios/Alternativas & Prioridades \\
\hline Programas Econômicos - PE & $\mathbf{0 , 7 1 7 0 6}$ \\
Programas Legais - PL & 0,21717 \\
Programas de Imagem - PI & 0,06577 \\
Reuso, revenda e reciclagem - A2, A3, A4 & 0,28222 \\
Custos por atividade de LR - A8, A9 & 0,49603 \\
Propaganda e novas tecnologias - A10, A11 & 0,06553 \\
Destino adequado aos resíduos - A12 & 0,07523 \\
Responsabilidade e cumprimento da lei - A20, A21 & 0,08099 \\
\hline
\end{tabular}

Fonte: elaboração própria (2021).

Percebe-se pela Tabela 4, que algumas observações podem ser feitas em relação ao ramo automobilístico. Foram destacados somente três programas como existentes na empresa. O programa econômico foi considerado o mais importante, seguido do programa legal.

Adicionalmente, a atividade A8 e A9, custos para operar o canal reverso e custos por desenvolver novas tecnologias relacionadas com o programa econômico, são os que têm maior importância.

Estes resultados mostram que o programa econômico ainda é o que possui maior importância, porém, o programa legal vem ganhando destaque, o que pode servir para uma comparação do panorama da LR antes e depois da PNRS. Desta maneira, é importante conhecer as práticas de LR que estão sendo utilizadas neste ramo, uma vez que a implementação da LR gera vários benefícios sociais. Os resultados podem servir para estruturar políticas públicas que fomentem o maior diálogo entre todos os agentes envolvidos, montadoras, recicladores, consumidores finais e governo. Adicionalmente, conhecer as práticas de LR no ramo automobilístico também podem atrair maiores investimentos, o que beneficia as empresas e o governo, de modo a aumentar o apoio governamental, ainda considerado pouco (Lima, 2016).

\section{Discussão dos Resultados}

Pode-se perceber que o programa legal quando comparado com outros estudos realizados em empresas brasileiras mostrou uma mudança significativa na importância. Quando comparado ao estudo de Hernández (2010), o programa econômico teve maior destaque. Entretanto, em grau de priorização, o programa legal não aparecia como um programa estabelecido para o ramo automobilístico, sendo o programa de cidadania, considerado o segundo mais importante. Entretanto, neste artigo, este se encontra em segundo lugar em termos de importância. Assim, nota-se que o programa legal teve sua relevância aumentada, o que pode indicar a importância da instalação da lei da PNRS.

Adicionalmente, tem-se o estudo de Leite et al. (2006), que inclui o ramo automobilístico nos ramos estudados. Neste estudo, o programa econômico também foi destacado, com 50\% de priorização, ao considerar quatro critérios, o econômico, a competitividade, a legislação e o meio ambiente, que incluiu aspectos de imagem e cidadania.

A Tabela 5mostra os critérios/programas priorizados no estudo de Hernández (2010) e neste artigo, para o ramo automobilístico em específico. 
Tabela 5. Prioridades dos programas de LR relacionadas a indústria automobilística no estudo de Hernández (2010) e neste artigo

\begin{tabular}{lcc}
\hline Critérios/Programas & $\begin{array}{c}\text { Vetor de prioridades (Hernandez } \\
\text { 2010) }\end{array}$ & $\begin{array}{c}\text { Vetor de prioridades (Neste } \\
\text { estudo) }\end{array}$ \\
\hline Programas econômicos - PE & 0,73064 & 0,71707 \\
Programas de imagem - PI & 0,08096 & 0,06577 \\
Programas de cidadania - & 0,18839 & Não relatado \\
PC & & 0,21717 \\
Progama Legal - PL & Não relatado & \\
\hline
\end{tabular}

Fonte: elaboração própria (2021).

Ao analisar a Tabela 5, nota-se que se mantêm três programas priorizados para este ramo. Entretanto, no estudo de 2010, os priorizados foram os critérios/programas econômicos, de imagem e de cidadania e no atual, o econômico, o legal e o de imagem. Nota-se que em 2010, o programa legal nem foi relatado como existente.

Em relação as atividades priorizadas no estudo de Hernández (2010), foi priorizado para o ramo automobilístico, a atividade de desenvolvimento de embalagens retornáveis e no atual estudo, as atividades relacionadas com coleta, seleção, transporte, armazenagem e o desenvolvimento de novas tecnologias e seus respectivos custos.

Em todo este cenário, a PNRS, homologada em 2010, preencheu uma lacuna na legislação brasileira ambiental baseado na gestão internacional e suas legislações. A principal mudança em relação a gestão dos resíduos se deu com o destaque da responsabilidade compartilhada pelo ciclo de vida do produto, acordos setoriais, LR, sustentabilidade operacional e financeira e envolvimento de padrões sustentáveis de produção e consumo (GRS-UFPE, 2014). Segundo esta lei, a LR seria parte importante para fazer o correto gerenciamento de resíduos e otimização do ciclo de vida dos produtos e estaria definida como um conjunto de procedimentos e atos que possam restituir os resíduos sólidos para as empresas (Luna \& Viana, 2019). Desta maneira, a LR, principal instrumento da PNRS, é importante na gestão de resíduos e em vários outros ramos, além do automobilístico.

Barbosa et al. (2019), destacam que gestores brasileiros na indústria de laticínio possuem conhecimento superficial quando se trata de desenvolvimento sustentável e citam como barreira principal, o alto custo de investimento. Esta barreira se enquadra nas principais dificuldades de implementação da PNRS, como falta de fundos governamentais, falta de capacidade técnica e administrativa, falta de conscientização do público em relação à geração de resíduos, falta de articulação da PNRS com outras políticas, falta de instrumentos de controle e cobrança e necessidade de maior articulação entre os entes federativos (Cetrulo et al., 2018; GRS-UFPE, 2014).

\section{Considerações Finais}

Ao analisar os resultados obtidos na seção 4, nota-se que o programa legal se torna mais relevante com a legislação brasileira cada vez mais rigorosa devido ao marco na gestão de resíduos, a PNRS. Entretanto, o Brasil ainda tem uma legislação defasada em relação a outros países.

O objetivo do artigo foi atingido ao se avaliar a importância dos motivadores de LR, no ramo automobilístico e na Região Sul Fluminense, após a implementação da PNRS. Percebe-se um maior destaque ao programa legal ao comparar esta pesquisa com a realizada 
por Hernández (2010). Este fato mostra que a questão da sustentabilidade ganha maior relevância, ao destacar como diferentes ramos da economia regional prioriza programas de LR e como estas fazem a gestão de seus resíduos sólidos. Neste cenário, práticas de LR mudaram depois da implementação da PNRS, uma vez que antes de 2010, faltava uma política que regulamentava e padronizava as tratativas no fim de vida útil dos produtos.

Kumar e Putnam (2008), destacaram algumas importantes atividades na implementação da LR na indústria automobilística tais como, reciclagem, reuso, revenda, custos para desenvolver novas tecnologias, destino adequado aos resíduos e envolvimento dos diversos stakeholders. Desta maneira, estas atividades se correspondem em grande parte com as encontradas no artigo que foram reuso, revenda e reciclagem, custos por atividades de LR, assim como para desenvolver novas tecnologias, destino adequado aos resíduos e cumprimento da legislação. De fato, os dois estudos se diferenciam nas atividades de envolvimento de diversos stakeholders, não encontrada no artigo e cumprimento da legislação, não encontrada no estudo de Kumar e Putnam (2008).

Como limitação, o estudo analisou uma região específica, a região Sul Fluminense e somente um ramo da economia, o automobilístico. A análise foi realizada com base nos questionários preenchidos por pessoas de determinadas empresas, o que confere subjetividade ao estudo, tendo o AHP função essencial em relação a este fator. Como recomendação de pesquisa futura, a análise pode ser feita em outras regiões e ramos, ou até mesmo na mesma região e ramos, mas em outras empresas para até mesmo ser feito um comparativo entre os estudos.

\section{Referências Bibliográficas}

Abbas, H., \& Farooquie, J. A. (2020). Reverse Logistics Practices in Indian Pharmaceutical Supply Chains: A Study of Manufacturers. International Journal of Logistics Systems and Management, 35(1), 72-89.

Agrawal, S., \& Singh, R. K. (2019). Analyzing Disposition Decisions for Sustainable Reverse Logistics: Triple Bottom Line Approach. Resources, Conservation and Recycling, 150(March), 104448.

Batista, L., Gong, Y., Pereira, S., Jia, F., \& Bittar, A. (2018). Circular Supply Chains in Emerging Economies - A Comparative Study of Packaging Recovery Ecosystems in China and Brazil. International Journal of Production Research, 7543.

Barbosa, B. B. C. C., Pereira, M. M. O., Antunes, L. G. R., Antonialli, L. M., \& Ferreira, M. C. (2019). A Inovação Ambiental em Laticínios Segundo seus Gestores: Um Estudo na Região do Alto Paranaíba-MG. Revista de Administração, Sociedade e Inovação, $5(3), 38-54$.

Bhandari, D., Singh, R. K., \& Garg, S. K. (2019). Prioritisation and Evaluation of Barriers Intensity for Implementation of Cleaner Technologies: Framework for Sustainable Production. Resources, Conservation and Recycling, 146(February), 156-167.

Beiler, B. C., Ignácio, P. S. A., Pacagnella Júnior, A. C., Anholon, R., \& Rampasso, I. S. (2020). Reverse Logistics System Analysis of a Brazilian beverage Company: An Exploratory Study. Journal of Cleaner Production, 274, 122624. 
Brito, M. P., \& Dekker, R. (2004). A Framework for Reverse Logistics. ERIM Report Series Research in Management, 3-27.

Cetrulo, T. B., Marques, R. C., Cetrulo, N. M., Pinto, F. S., Moreira, R. M., Mendizábal-Cortés, A. D., \& Malheiros, T. F. (2018). Effectiveness of Solid Waste Policies in Developing Countries: A Case Study in Brazil. Journal of Cleaner Production, 205, 179187.

CNI. (2019). Economia Circular: Caminho Estratégico para a Indústria Brasileira. 68.

Cosenza, J. P., Andrade, E. M. D., \& Assunção, G. M. De. (2020). A Circular Economy as an Alternative for Brazil's Sustainable Growth: Analysis of the National Solid Waste Policy. Revista de Gestao Ambiental e Sustentabilidade, 9(1), 1-30.

Couto, M. C. L., \& Lange, L. C. (2017). Análise dos Sistemas de Logística Reversa no Brasil. Engenharia Sanitaria e Ambiental, 22(5), 889-898.

Creswell, J. W. (2007). Projeto de Pesquisa: Métodos Qualitativo, Quantitativo e Misto. $2^{a}$ edição,Porto Alegre: Artmed.

Demajorovic, J., Huertas, M. K. Z., Boueres, J. A., Silva, A. G., \& Sotano, A. S. (2012). Logística Reversa: Como as Empresas Comunicam o Descarte de Baterias e Celulares? Revista de Administração de Empresas, 52(2), 165-178.

Elkington, J. (1998). Accounting for the Triple Bottom Line. Measuring Business Excellence, 2(3), 18-22.

Eltayeb, T. K., Zailani, S., \& Filho, W. L. (2010). Green Business Among Certified Companies in Malaysia Towards Environmental Sustainability: Benchmarking on the Drivers, Initiatives and Outcomes. International Journal of Environmental Technology and Management, 12(1), 95-125.

Escrig-Olmedo, E., Fernández-Izquierdo, M. A., Ferrero-Ferrero, I., Rivera-Lirio, J. M., \& Muñoz-Torres, M. J. (2019). Rating the Raters: Evaluating how ESG Rating Agencies Integrate Sustainability Principles. Sustainability (Switzerland), 11(3).

Farooque, M., Zhang, A., Thürer, M., Qu, T., \& Huisingh, D. (2019). Circular Supply Chain Management: A Definition and Structured Literature Review. Journal of Cleaner Production, 228(April), 882-900.

Ferreira, N. de M., Santos, L. C., \& Silva, M. L. P. da. (2021). Stakeholders e o Gerenciamento de Coprodutos: Logística Reversa e Simbiose Industrial na Redução de Custos. Brazilian Journal of Development, 7(3), 33182-33201.

Geissdoerfer, M., Savaget, P., Bocken, N. M. P., \& Hultink, E. J. (2017). The Circular Economy - A New Sustainability Paradigm? Journal of Cleaner Production, 143, 757-768.

Geisendorf, S., \& Pietrulla, F. (2018). The Circular Economy and Circular Economic Concepts - A Literature Analysis and Redefinition. Thunderbird International Business Review, 60(5). 
Geng, Y., Fu, J., Sarkis, J., \& Xue, B. (2012). Towards a National Circular Economy Indicator System in China: An Evaluation and Critical Analysis. Journal of Cleaner Production, 23(1).

Gerhardt, T. E., Silveira, D. T. (2009). Métodos de Pesquisa. Universidade Aberta do Brasil - UAB/UFRGS. Porto Alegre: Editora da UFRGS.

Ghisellini, P., Cialani, C., \& Ulgiati, S. (2016). A Review on Circular Economy: The Expected Transition to a Balanced Interplay of Environmental and Economic Systems. Journal of Cleaner Production, 114, 11-32.

Gil, A. C. (2007). Como Elaborar Projetos de Pesquisa. 6a edição, São Paulo: Atlas.

Govindan, K., \& Bouzon, M. (2018). From a Literature Review to a Multi-Perspective Framework for Reverse Logistics Barriers and Drivers. Journal of Cleaner Production, 187, 318-337.

GRS-UFPE. (2014). Análise das Diversas Tecnologias de Tratamento e Disposição Final de Resíduos Sólidos Urbanos no Brasil, Europa, Estados Unidos e Japão. 188. http://www.bndes.gov.br/SiteBNDES/export/sites/default/bndes_pt/Galerias/Arquivos/produt os/download/aep_fep/chamada_publica_residuos_solidos_Relat_Final.pdf

Hernández, C. T. (2010). Modelo de Gerenciamento da Logística Reversa Integrado às Questões Estratégicas das Organizações.

Hernández, C. T., Marins, F. A. S., \& Castro, R. C. (2012). Modelo de Gerenciamento da Logística Reversa. Gestao e Produçãoo, 19(3), 445-456.

Hernández, C. T., Marins, F. A. S., Rocha, P. M., \& Durán, J. A. R. (2010). Using AHP and ANP to Evaluate the Relation Between Reverse Logistics and Corporate Performance in Brazilian Industry. Brazilian Journal of Operations \& Production Management Volume, 7(2), 47-62.

Jonathas, R., \& Paiva, A. D. (2020). O Cluster Automotivo Sul Fluminense. 10-23.

Kumar, S., \& Putnam, V. (2008). Cradle to Cradle: Reverse Logistics Strategies and Ppportunities Across Three Industry Sectors. International Journal of Production Economics, 115(2), 305-315.

Lang, D. J., Wiek, A., Bergmann, M., Stauffacher, M., Martens, P., Moll, P., Swilling, M., \& Thomas, C. J. (2012). Transdisciplinary Research in Sustainability Science: Practice, Principles, and Cchallenges. Sustainability Science, 7(SUPPL. 1), 25-43.

Leite, P. R. (2012). Direcionadores Estratégicos Em Programas de Logística Reversa no Brasil. Revista Alcance, 19(2), 182-201. https://doi.org/10.14210/alcance.v19n2.p182-201.

Leite, P. R., De Brito, E. P. Z., Macau, F., \& Povoa, Â. (2006). O Papel dos Ganhos Econômicos e de Imagem Corporativa na Estruturação dos Canais Reversos. Revista Eletrônica de Gestão Organizacional, 5-21.

Lima, N. N. H. (2016). Logística Reversa no Setor Automobilístico Brasileiro: Uma 
Aplicação para o Estado de São Paulo.

Luna, R. A., \& Viana, F. L. E. (2019). O Papel da Política Nacional dos Resíduos Sólidos na Logística Reversa em Empresas Farmacêuticas. Revista de Gestão Social e Ambiental, 13(1), 40-56.

Machado, H. H. D. S., Sgorlon, J. G., Altoé, S. P. S., Meneguetti, K. S., Oliveira, J. C. D., Martins, C. H., \& Tavares, C. R. G. (2012). A Gestão dos Resíduos Sólidos Industriais Aplicadas a Países Desenvolvidos e em Desenvolvimento. Congreso Latino-Americano de Ecologia Urbana.

Mangla, S., Madaan, J., \& Chan, F. T. S. (2012). Analysis of Performance Focused Variables for Multi-Objective Flexible Decision Modeling Approach of Product Recovery Systems. Global Journal of Flexible Systems Management, 13(2), 77-86.

Mota, M. C. S. A. R. C., \& Paula, E. V. (2020). Identifying Barriers and Proposing a Roadmap to Develop Industrial Symbiosis Projects. Revista Administração da UFSM, 11(2), 471-488.

Ness, D. (2008). Sustainable Urban Infrastructure in China: Towards a Factor 10 Improvement in Resource Productivity through Integrated Infrastructure Systems. 15, 288301.

Oliveira, C. T., Luna, M. M. M., \& Campos, L. M. S. (2019). Understanding the Brazilian Expanded Polystyrene Supply Chain and its Reverse Logistics Towards Circular Economy. Journal of Cleaner Production, 235, 562-573.

Oliveira, F. R., França, S. L. B., \& Rangel, L. A. D. (2018). Challenges and Opportunities in a Circular Economy for a Local Productive Arrangement of Furniture in Brazil. Resources, Conservation and Recycling, 135(January), 202-209.

Pinheiro, A. B., Chagas, A. C. C., Santos, S. M. S., Silva, S. M., \& Lopes, F. M. D. A. (2020). Obstáculos para a Adoção da Simbiose Industrial no Distrito Industrial de Maracanaú: Insights para o Desenvolvimento Sustentável. Revista de Casos e Consultoria, 1-24.

Saaty, T. L., \& Sodenkamp, M. (2010). The Analytic Hierarchy and Analytic Network Measurement Processes: The Measurement of Intangibles. 1(1), 91-166. https://doi.org/10.1007/978-3-540-92828-7_4

Su, B., Heshmati, A., Geng, Y., \& Yu, X. (2013). A Review of the Circular Economy in China: Moving from Rhetoric to Implementation. Journal of Cleaner Production, 42, 215227.

Trevisan, M., Nascimento, L. F., Madruga, L. R. da R. G., Neutzling, D. M., Figueiró, P. S., \& Bossle, M. B. (2016). Ecologia Industrial, Simbiose Industrial e Ecoparque Industrial: Conhecer para Aplicar. Sistemas \& Gestão, 11(2), 204-215.

Vlachos, I. P. (2016). Reverse Logistics Capabilities and Firm Performance: The Mediating Role of Business Strategy. International Journal of Logistics Research and Applications, 19(5), 424-442. 
Vieira, B. O., Guarnieri, P., Silva, L. C., \& Alfinito, S. (2020). Prioritizing Barriers to be Solved to the Implementation of Reverse Logistics of e-Waste in Brazil under a Multicriteria Decision aid Approach. Sustainability (Switzerland), 12(10).

Wang, J., \& Wang, Z. (2020). Strengths, Eeaknesses, Opportunities and Threats (Swot) Analysis of China's Prevention and Control Strategy for the Covid-19 Epidemic. International Journal of Environmental Research and Public Health, 17(7). 